\title{
Perspectives on the Design, Challenges, and Evaluation of Smart TV User Interfaces
}

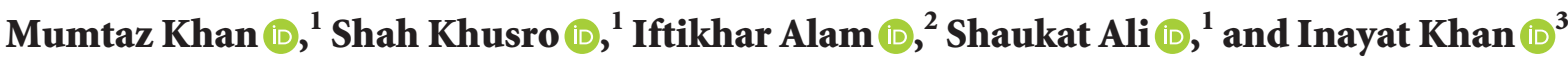 \\ ${ }^{1}$ Department of Computer Science, University of Peshawar, Peshawar, 25010, Pakistan \\ ${ }^{2}$ Department of Computer Science, City University of Science and Information Technology, Peshawar, 25010, Pakistan \\ ${ }^{3}$ Department of Computer Science, University of Buner, Buner, Pakistan \\ Correspondence should be addressed to Shah Khusro; khusro@uop.edu.pk
}

Received 22 October 2021; Revised 3 January 2022; Accepted 28 January 2022; Published 23 February 2022

Academic Editor: Rahman Ali

Copyright (c) 2022 Mumtaz Khan et al. This is an open access article distributed under the Creative Commons Attribution License, which permits unrestricted use, distribution, and reproduction in any medium, provided the original work is properly cited.

\begin{abstract}
The user interface (UI) is a primary source of interaction with a device. Since the introduction of graphical user interface (GUI), software engineers and designers have been trying to make user-friendly UIs for various computing devices, including smartphones, tablets, and computers. The modern smart TV also comes with built-in operating systems. However, little attention has been given to this prominent entertainment device, i.e., smart TV. The technological advancement and proliferation of smart TV enabled the manufacturer to provide rich functionalities and features; however, this richness resulted in more clutter and attention-demanding interfaces. Besides, smart TV is a lean-back supporting device having a diverse range of users. Therefore, smart TV's usability and user experience (UX) are questionable due to diverse user interests and limited features of traditional remote controls. This study aimed to discuss and critically analyze the features and functionalities of the existing well-known smart TV UIs of various operating systems in the context of usability, cognition, and UX. Moreover, this study highlights the issues and challenges in the current smart TV UIs and recommends some research opportunities to cope with the smart TV UIs. This study further reports and validates some overlooked factors affecting smart TV UIs and UX. A subjective study and usability tests from diverse users are presented to validate these factors. The study concludes that a one-size-fits-all UI design is unsuitable for shared devices, i.e., smart TV. This study further recommends a personalized adaptive UI, which may enhance the learnability and UXs of the smart TV viewers.
\end{abstract}

\section{Introduction}

The user interface (UI) is an essential, dominant, and primary source of communication with a device and enables a user to perform various operations to access device functionalities, features, and services. Nowadays, the UI of different interactive systems, including smartphones, tablets, smart TVs, and computers, provides an enriched set of functionalities and features. However, most users cannot utilize all these functions properly due to complexity, clutter, and poor UI design [1]. A smart TV is a lean-back and shared device for individuals and family members [2]. Various applications have been developed for smart TVs that provide multiple services and functionalities [2-4], such as video-on-demand services, online and offline games, live channels, movies, dramas, TV shows, Web browsing, social networking, and recommendation. The latest smart TVs are also embedded with branded operating systems. However, little attention has been given to this prominent entertainment device, i.e., smart TV [5]. According to a researchand-market report, a smart TV unit is expected to increase from around 209.3 million in 2019 to 266.4 million in 2025, with a compound annual growth rate of 4.1 percent [6]. Moreover, the US Bureau of Labor Statistics reported that in 2020, most Americans spent leisure time watching TV [7]. This incredible growth rate shows that each household will have a smart TV soon [8-10].

A smart TV provides a diverse set of contents from numerous sources through various applications (apps in short), and each app offers specific functionalities and 
services to the users [11]. These applications are developed for domain-specific purposes and used by various viewers, such as children, senior citizens, and technical and nontechnical viewers. However, all types of viewers cannot use all the available apps. Moreover, each user's interaction and watching behavior are different due to age and gender differences, skills, viewing distance, interaction behavior, screen size, cultural values, and mental model. The activities on a smart TV, such as watching content, viewing behavior, searching behavior, user interaction behaviors, and interaction modalities, are different from smartphones and tablets [12]. The integration of Web 2.0 features, processing capabilities, Internet connectivity, operating systems, thirdparty platforms, and media players come with smart TV made this device attractive for the end users and researchers $[3,4,8,11]$.

The diversification of the contents and advanced functionalities of a smart TV added more to enjoyment. However, such diversity and enrichments of features bring more complexities in UIs $[13,14]$. In contrast, accessing and switching channels on traditional TV through remote control were not a cumbersome job [13]. The new emerging types of activities with smart TVs, such as installation of apps, channel selection, app updating, closing, navigation between inter- and intra-app, app deleting, assigning priority, app management, create complexities and hurdles for viewers and bring the issues in the form of learnability, usability, and cognitive overload [15]. Although smart TV is accessed and operated by various interaction modalities, such as remote controls, small keypad, voice, gestures, virtual keyboard, and smartphone-based apps, still, most of the viewers use the traditional remote control as a primary interaction device that limits frequent operations and interactions [12].

The current smart TV UIs have static capabilities containing various issues and problems, such as navigation issues, content searching and browsing issues, channel switching issues, and scrolling issues. Furthermore, viewers cannot change the UIs, content organization, menu structure, and style of the smart TV UI to enhance usability and better UX. Thus, the researchers, manufacturers, and vendors have given less attention to developing personalized and adaptive user interfaces that are acceptable and can fulfil various smart TV viewers' requirements $[16,17]$. The smart TV vendors and manufacturers have developed various smart TV operating systems and home screen launchers, i.e., Android TV, WebOS, Tizen, tvOS, and Roku TV OS. Each vendor and manufacturer of smart TV is trying to develop and redesign market-oriented hardware and operating systems for their smart TVs to attract viewers and customers and gain a high market share. In this race, the vendors and companies regularly update and enrich the features and functionalities of their operating systems, home screen launchers, and hardware [15]. However, this extra day-byday enrichment of functions and features in smart TV operating systems creates usability issues, unpleasant UX, cognitive overload, and learnability issues $[14,18]$. According to the Statista Research Department report, in 2018, Android was the world's leading smart TV operating system with a market share of about $40 \%$. This Google's popular platform was dominant compared with its closest competitor, Tizen, by around $17 \%$, respectively, and so on [19]. The functionalities and features of each operating system have been critically analyzed (see Related Work section). These advanced features and functionalities of smart TV operating systems can make smart TV more intelligent for the viewers according to their needs and preferences.

Different approaches such as online survey, thinkingaloud methods [11, 20], model-based automated approach, dairy approach, user-centered approach [21], analytical approach, and laboratory approach have been used to evaluate consumer usage habits, attitudes, and interactions to gather comprehensive data from smart TV UX [14, 22] for the enhancement of user interfaces. However, smart TV is an emerging device with complex interactions and supports various functions and features [4].

Besides, the manufacturers of smart TV have designed different UIs that facilitate the users to interact with and navigate between the available contents. However, different UIs make interaction a hectic and challenging task for the users [13]. The interactions and usage of each viewer with the smart TV are different from one another due to the mental model, age, gender, knowledge level, nature, cultural values/ dimensions, attitudes, and disabilities. In this connection, various approaches, methods, and guidelines have been proposed to overcome the usability and accessibility issues of smart TV [13, 23-26]. Still, they usually focused on solving UI's general problems for all users and not designing and creating adaptivity in the UI for a specific user because the perspective, preferences, privacy, learnability, and cognition capabilities of each user are different in terms of a mental model, usage, interaction behavior, knowledge, skills, gender, age, educational level, and cultural values. For example, senior citizens need relevant apps, so unnecessary UI things can be removed/hidden. They need simple UI to overcome their cognitive overload, learnability, and usability issues and hide the excessive and irrelevant apps that are not suitable for them. Similarly, the requirements and preferences of housewives are different from other family members, such as kids. An app watched by one person may not be relevant to other viewers and may leak privacy issues.

The contributions of this study are as follows:

Comparison of various features and functionalities of different well-known smart TV operating systems and their UIs

Critical investigation of issues and challenges in existing well-known smart TV UIs

Highlighting the factors that affect the usability and UX of the smart TV UIs

Subjective study for analyzing the smart TV UIs

The results of the study show that each household has a diverse range of users regarding taste, nature, preferences, needs, age, skills, knowledge, attitude, learnability, and UX. It is also a fact that most viewers do not use all features and functionalities of smart TV. The watching and interaction 
behavior of the household viewers are different from one another due to various factors (as discussed). Moreover, some viewers did not welcome the enrichments of new features and functions in smart TV that may lead the usability and UX issues in the smart TV user interface. The findings of this study recommend that the existing smart TV user interfaces of various brands and companies treat all the household viewers with the exact nature, knowledge, taste, age, preferences, needs, skills, cultural values, etc. The other computing devices, such as computers and smartphones, are personalized devices, whereas smart TV is shared. Furthermore, an adaptive user interface may play a key role in smart TV UIs and enhance smart TV's usability and UX. This study further suggests a personalized adaptive user interface according to the viewer's needs, preference, age, mental model, cultural value, and environmental variability to enhance the user usability and UX in a smart TV viewing environment.

The rest of the study is organized into seven sections. Section 2 discusses the state-of-the-art literature on usability and UX in the context of the smart TV UI. Section 3 offers the usability issues and challenges that affect the usability and UX of the smart TV user interfaces for the viewers. Section 4 discusses the overlooked factors that affect usability and UXs of the smart TV UIs for the viewers. Section 5 presents the factor validation. Similarly, Section 6 discusses the results and analysis. Section 7 is a discussion, and Section 8 concludes the study and discusses future research work.

\section{Related Work}

Television (TV in short) is an important entertainment source and plays a central role in the home environment [27]. Watching TV is one of the most common habits worldwide, and most people spend their leisure time accessing news, dramas, and movies [28]. According to the survey report $[28,29]$, TV is still one of the most popular entertainment devices globally. The daily watching consumption is high from the collective activities on smartphones, tablets, and computers. However, traditional TV only provides broadcast channels with passively consumed medium with limited interaction. In addition, TV was used as a watching medium, and interaction of users was to change channels and programs through remote control. The concept of watching behavior and using traditional TV is altered due to the smart TV and technologically advanced features and functionalities. Today, smart TV provides a variety of services, including processing, storage, connectivity, Web 2.0 features, embedded sensors, peripheral device support, social networking, offline and online gaming, news, TV shows, movies, Web browsing, face recognition, automatic content recognition, motion control, and voice search via remote and smartphone-based apps $[4,12,15]$. Besides, smart TV comes with various operating systems, UIs, home screen launchers, third-party apps, middleware, and various development platforms. Smart TV has become one of the most popular entertainment devices and has attracted viewers and researchers [11]. On the other hand, smart TV provides app-based entertainment, services, and various programs to the user, which is not in the scope of traditional TV.
For technological advancement, the vendors and manufacturers have developed various smart TV operating systems and home screen launchers/launchpads, i.e., Android TV, WebOS, Tizen, tvOS, and Roku TV OS. Each vendor and manufacturer of smart TV is trying to develop, upgrade, change, and redesign market-oriented hardware and operating systems for their smart TVs to attract viewers and customers and gain high market value. In this competition, the vendors and companies are trying to update and embed the new features and functionalities to the operating systems, home screen launchers, and hardware [15]. However, this regular updating and embedding of the latest advanced functions and features in the smart TV operating system create usability, UX, cognitive overload, and learnability of the smart TV UIs [18]. Companies and vendors treat smart TVs such as smartphones and tablets; however, it is a shared device used by various users. The smart TV UIs are in the infant stage, and the researcher is given little attention to usability factors and UX.

Nowadays, the user interfaces of various well-known smart TVs provide an enriched set of functionalities and features. Still, most users cannot properly utilize their functions due to a complex and cluttered user interface [1]. The designers of user interfaces face numerous problems and issues, including different computing platforms, interaction modalities, input/output skills of viewers, markup languages, developing toolkits, and contextual variability [5]. Therefore, handling various users' requirements is challenging through a single "one-size-fits-all" user interface [30]. Furthermore, it ignores the accessibility and usability requirements for diverse user groups such as kids, adults, senior citizens, and technical and nontechnical users. Additionally, the development and design of multiple UIs for user groups that provide various functionalities and features are a time-consuming and challenging task to handle the context variability at the design time [31-33]. The subsections discuss the different components, including operating systems, features, and UIs of different smart TVs.

\subsection{State-of-the-Art Smart TV Operating Systems and UIs.} The smart TV operating system manages and controls the hardware and software. It also provides access and various services to the users to perform different operations and control advanced features and connected devices, such as watching channels, TV shows, video-on-demand services, movies, news, dramas, gaming, processing, storage, screen casting, home automation, searching and browsing, automatic content recognition, and Web 2.0 features [8]. According to the market report, the most common, popular, and state-of-the-art operating systems of the smart TV are Android TV, WebOS, Tizen, tvOS, and Roku TV OS $[8,15]$. The brief introduction of each smart TV operating system and its UI is discussed in the following subsection, and their various features and functions are summarized in Tables 1 and 2. In addition, the features and functionalities of each operating system are enlisted to show the usability and UX issues of a user interface in the context of the smart TV watching scenario. 
TABLE 1: Common features and functionality comparison of smart TV operating system.

\begin{tabular}{lcccccc}
\hline \multirow{2}{*}{ Operating system } & & \multicolumn{2}{c}{ Common features } \\
& Google assistant & Voice search & Content recommendation & Screen casting & Channel customization & Smart home \\
\hline Android & $\checkmark$ & $\checkmark$ & $\checkmark$ & $\checkmark$ & $\checkmark$ & $\checkmark$ \\
WebOS & $\checkmark$ & $\checkmark$ & $\checkmark$ & $\checkmark$ & $\checkmark$ & $\checkmark$ \\
Tizen & $\checkmark$ & $\checkmark$ & $\checkmark$ & $\checkmark$ & $\checkmark$ & $\checkmark$ \\
tvOS & $\checkmark$ & $\checkmark$ & $\checkmark$ & $\checkmark$ & $\checkmark$ \\
Roku OS & $\checkmark$ & $\checkmark$ & $\checkmark$ & $\checkmark$ & $\times$ \\
\hline
\end{tabular}

2.1.1. Android TV. Google developed an Android (https:// www.android.com/tv/) operating system for smart TVs and was first announced in the annual conference of Google I/O in June 2014 as the successor of Google TV. It is an opensource operating system based on the Linux kernel. It is developed to operate digital media players, set-top boxes, and smart TVs. Most brands, vendors, and companies use Android TV operating systems for smart TVs, set-top boxes, and Android TV sticks. According to the Statista Research Department report in 2018, Android was the world's leading smart TV operating system with a market share of about $40 \%$. This Google's popular platform outperformed its closest competitor, i.e., Tizen by around 17\% [19]. The operating system versions of Android TV are regularly updating, redesigning, and embedding the new features and functionalities to attract customers and market shares worldwide. However, these enrichments of features create usability and UX in the context of smart TV user interfaces. Besides, various vendors and companies are using Android TV operating systems. This diversification of user interfaces can create the issue of usability and UX for the viewers. The latest version of the Android TV operating system is Android TV 10. The detailed characteristics and functionalities of the Android TV 10 version are shown in Tables 1 and 2.

2.1.2. WebOS. The WebOS (http://webostv.developer.lge. com/discover/discover-webos-tv/) is developed by LG Electronics for smart TV, an open-source platform based on Linux kernel. Like the Android TV operating system, it is used by various vendors and companies for smart TVs, settop boxes, and dongles. This operating system is especially well known for its speed; however, it is not easily customizable. It provides various services, features, and functionalities to the viewers depicted in Tables 1 and 2. With the advent of technological advancement, the parts and functionalities of WebOS are regularly updating, redesigning, and adding new features in the OS to attract viewers and gain high market value in the world. Therefore, these changes may create the issue of usability and UXs in the viewers. The latest version of the LG smart TV is WebOS TV 5.0. The detailed features and functionalities of WebOS TV 5.0 are shown in Tables 1 and 2.

2.1.3. Tizen OS. The Tizen (https://www.tizen.org/) is an open-source operating system developed by Samsung and used mainly by Samsung's smart devices, including smart TV. It provides full-pledge support for native applications developed for smart devices. Like other smart TV operating, it is also used by various vendors and companies for the smart TV, set-top boxes, and dongles. The Tizen OS also provides various services, features, and functionalities to the viewers depicted in Tables 1 and 2 . The latest version of the Samsung smart TV is Tizen 5.5 TV OS.

2.1.4. $t v O S$. The tvOS (https://developer.apple.com/tvos/) is an operating system that operates Apple TVs and digital media players developed by Apple. It is based on the iOS and adapts many similar features, technologies, concepts, and frameworks of the iOS. The initial generations of tvOS, including 2 nd and 3rd, consist of several built-in applications but do not support third-party apps. The other generations of tvOS, including 4th and upward, support the capabilities of the third-party apps. Like other smart TV operating systems, it regularly updates, redesigns, and embeds new features and functionalities to the tvOS to maintain the business status and attract viewers. The latest version of the tvOS 14 has adopted many parts of the iOS 14 operating system, such as picture-in-picture to third-party apps, HomeKit, redesigned the control center. The tvOS also provides various services, features, and functionalities to the viewers depicted in Table 1 . The latest version of the Apple smart TV operating system is tvOS 14 .

2.1.5. Roku TV OS. Roku (https://www.roku.com/) OS is a smart operating TV developed by Roku. It is specially designed to work with smart TVs, digital media players, dongles, and soundbars. The popularity of the Roku TV platform is increasingly growing in the USA. According to the statistics of Statista (https://www.statista.com/statistics/ 1021332/united-states-connected-tv-devices-market-share/ ), the streaming TV platform of the Roku Company in the first quarter of 2019 is about $30 \%$ of all connected TV device sales in the USA. In the first quarter of 2019, Roku reported close to 30 million monthly active users in the USA. The annual revenue of Roku was over 325 million US dollars in 2018. It is an open-source platform and is based on Linux operating system. In addition, it is also used by various vendors and companies for smart TVs, set-top boxes, and dongles. It provides multiple services, features, and functionalities to the viewers, depicted in Tables 1 and 2. The features and functionalities of Roku OS are regularly updating, redesigning, and adding new features in the OS to attract viewers and gain high market value in the world. These changes may create the issue of usability and UXs in the viewers. The latest version of the Roku OS of smart TV is Roku OS 9.3. 
TABLE 2: Uncommon features and functionality comparison of smart TV operating system.

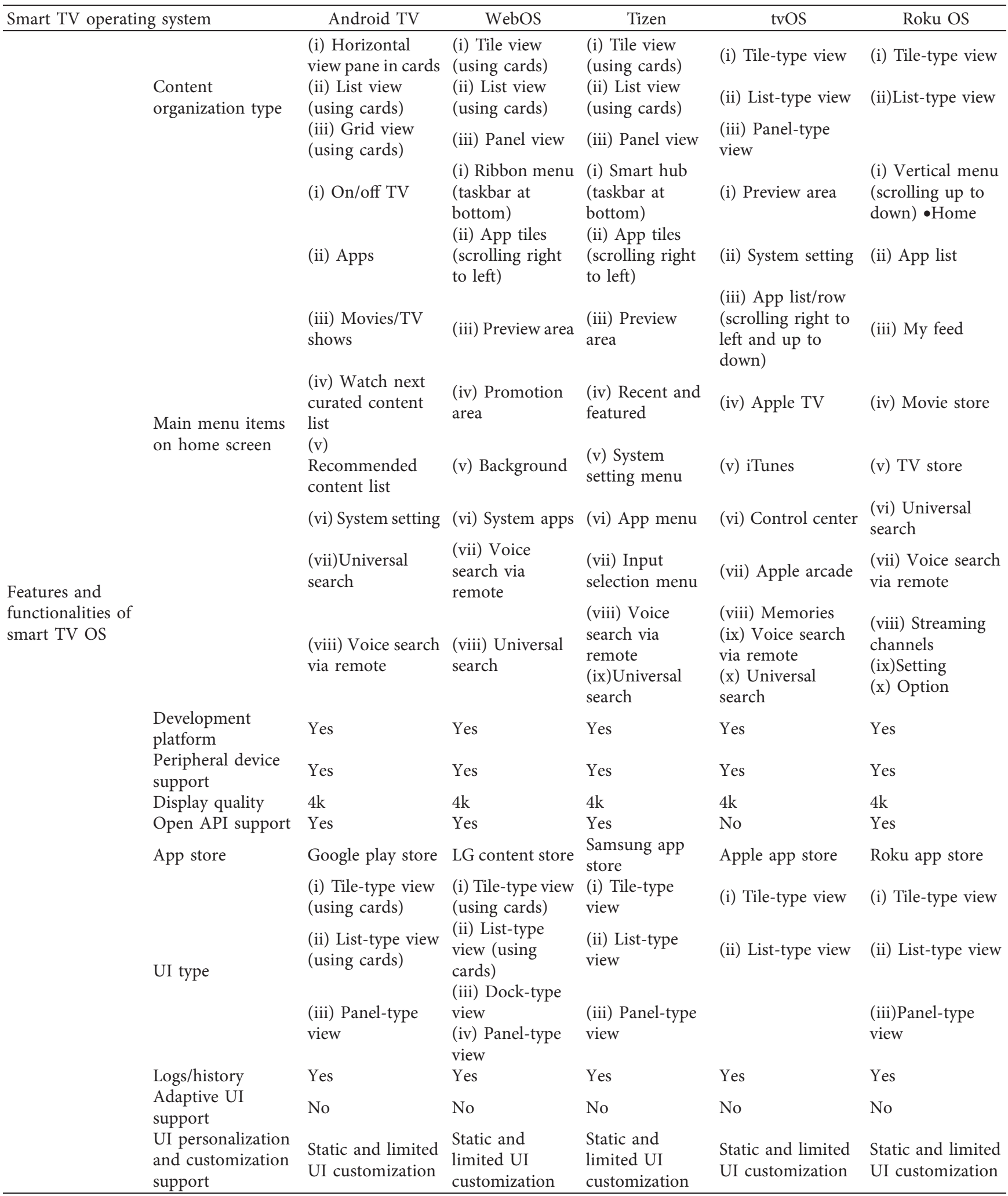

2.1.6. Smart TV User Interfaces. Today's smart TV operating systems have built-in user interfaces (UIs) that enable the viewers to interact with a smart TV. In addition, it enables the viewers to perform various operations and tasks on smart TV. The collection of fundamental operations/tasks on the smart TV user interface is summarized in Table 3. These tasks/operations are separately tested on each smart TV user interface to investigate the different dimensions of 
TABLE 3: Smart TV UI summary.

\begin{tabular}{lccc}
\hline S. No. & Function/operation/task & & Interaction method \\
& & Remote control & $\checkmark$ \\
Keyboard & $\checkmark$ \\
2 & On/off smart & $\checkmark$ & $\checkmark$ \\
3 & User profile setting & $\checkmark$ & $\checkmark$ \\
4 & Account switching & $\checkmark$ & $\checkmark$ \\
5 & Search for contents & $\checkmark$ & $\checkmark$ \\
6 & Browsing the contents through browsers & $\checkmark$ & $\checkmark$ \\
7 & Social networking & $\checkmark$ & $\checkmark$ \\
8 & App open and close & $\checkmark$ & $\checkmark$ \\
9 & Search for channels & $\checkmark$ & $\checkmark$ \\
10 & Channel switching & $\checkmark$ & $\checkmark$ \\
11 & Channel management & $\checkmark$ & $\checkmark$ \\
12 & App management & $\checkmark$ & $\checkmark$ \\
13 & Screen mirroring/casting & $\checkmark$ & $\checkmark$ \\
14 & App installation and uninstallation & $\checkmark$ & $\checkmark$ \\
15 & Online and offline gaming & $\checkmark$ & $\checkmark$ \\
16 & System setting and connection & $\checkmark$ & $\checkmark$ \\
17 & Navigation intra-apps & $\checkmark$ & $\checkmark$ \\
18 & Navigation inter-apps & $\checkmark$ & $\checkmark$ \\
19 & Scrolling up and down & $\checkmark$ & $\checkmark$ \\
\hline
\end{tabular}

the various viewers, including children, homemakers, adults, technical and technical, educated and noneducated, and senior citizens.

The vendors and companies regularly update, change, redesign, and embed new features and functionalities in the hardware operating system to attract customers and gain high market revenue. However, this day-by-day enrichments of advanced parts and functionalities can made the user interface clutter and complex for the viewers. However, a smart TV is a shared device used by family members. Each family member's chrematistics is different from one another, such as age, skill, mental model, gender, preference, needs, cultural dimension, attitudes, and disabilities. Tables 1 and 2 represent various features and functions of smart TV operating system, including features, content organization type, main menu item on the home screen, development platform, peripheral device support, display quality, open API support, app store, user interface (UI) type, interaction devices, log/history, adaptive UI support, and UI customization and personalization support. In Tables 1 and 2, we can conclude that none of the existing smart TV operating systems support an adaptive user interface according to viewers' needs, preferences, age, gender, skill, and cultural values. In addition, the available state-of-the-art user interfaces of smart TVs are designed for all users that contain a rich set of features, which do not handle the issues of usability and UX and cannot satisfy the requirements of each individual according to their needs, usages, age, gender, knowledge and mental model, cultural values/dimensions, attitudes, and disabilities. The following section will investigate some overlooked factors that affect usability and user. The users are still performing basic operations/tasks through primary interactive devices. The researchers have used various approaches such as model-driven [34, 35], userdriven/user-centered design [36, 37], context-driven [38], ontology-based $[39,40]$, and machine learning-based
$[41,42]$ to overcome the usability and UX issues of complex user interfaces of the interactive systems and proposed the concept of adaptive user interfaces to fulfil the requirements of each individual according to their abilities, preference, ages, mental mode, skills, and education. Adaptive user interfaces have been used in many domains, including operating systems, Web-based applications, and smartphone-based apps [43]. The primary purpose of the adaptive user interface is to overcome the usability and accessibility issues and provide tailored assistance to the users according to their needs and preferences [32]. Besides, it enhances the interaction between the user and the system. The adaptive user interface adjusted the layouts, elements, components, font size, color, contrast, theme, and app arrangements according to the user's requirements, abilities, experiences, and context [41]. The adaptive user interfaces reduce the usability issues and challenges of complex and cluttered user interfaces and improve the users' performance $[34,41]$.

Various adaptive user interfaces have been developed for senior citizens, children, drivers, blind and visually impaired person, and domain-specific applications, such as e-commerce applications (eBay, Amazon), health applications, gamification applications, e-learning applications, and entertainment applications [32, 41, 44-53] for various computing platforms, i.e., smart TVs, smartphones, and computers. However, the intelligent TV UIs are in the infant stage. It cannot focus on the user's characteristics and environmental factors with different contexts, such as light, color, noise, time of the day, and weather.

Different approaches are used for the enhancement of user interfaces. However, smart TV is an emerging device with complex interactions and supports various functions and features. The manufacturers of smart TV have designed different UIs that facilitate the users to interact with to navigate between the available contents. Still, they do not fulfil the requirements of each individual. Furthermore, each 
manufacturer's UI is different, creating interaction hurdles and confusion for the users [13]. The interactions and usage of everyone with the smart TV are different from one another due to the mental model, age, gender, knowledge level, nature, cultural values/dimensions, attitudes, and disabilities. However, the current start-of-the-art UIs of different manufacturers are far from handling each individual's needs and perspective.

Moreover, various methods and guidelines have been proposed to overcome the usability, UX, and accessibility issues of smart TV [13, 14, 23-26]. Still, they usually focused on solving UI's general problems for all users and not designing and creating adaptivity in the UI for a specific user. For example, kids need those relevant apps to their level and do not need unnecessary UI things. Similarly, the requirements and preferences of homemakers are different from other family members. Furthermore, senior citizens' needs and watching behavior are also different from other household users. Similarly, children have diverse tastes and preferences from other household members. The following section reports the usability barriers in smart TV UIs.

\section{Usability Issues in Smart TV UIs}

This section will discuss some potential factors/issues and challenges of the existing smart TV user interface in usability, UX, learnability, accessibility, and cognitive overload. These factors recommend that the smart TV user interface ignores various parameters such as age, gender, skills, technical and nontechnical, senior citizen, and cultural values.

3.1. Channel Inter-Intra Navigational Complexities. The smart TV's app developers have designed and developed various UIs. Besides, some apps are engineered and treated like smartphones or tablets, and developers follow the similar design style. However, smart TV is different from smartphones, tablets, and even computers in terms of interaction, user type, shared nature, viewing style, viewing distance, viewing angle, etc. In contrast to smart TV, traditional TV provides a limited number of terrestrial channels in which navigation is an easy task through remote control and does not create usability, learnability, and cognitive overload for the viewers [8]. However, smart TV provides diverse content sets for watching and interactions from various sources through apps such as entertainment, talk show, games, and social networking. Besides, content providers have developed different apps, and each app has a diverse set of functions and features for interactions. The smart TV accesses the contents through apps, creating interand intra-app navigation hurdles. Moreover, the smart TV interface becomes cluttered for the viewers [54]. Other reasons for navigation issues in the UI of smart TV include viewing distance, viewing angle, screen size, and resolution. These factors can cause UI interaction issues for viewers. The viewers interact with UI from a standard distance with the help of remote devices. Usually, the average distance for smart TV viewing is 8 to 10 feet. Besides, smart TV manufacturing includes extra features and functionalities, including Google assistant, ThinQ AI, and smart hub that make UIs complex to use [55].

3.2. User Interface Issues. The manufacturers, vendors, and companies have developed various user interfaces for smart TVs. Each company adds new features and functionalities to the smart TV to attract customers and gain a high market share worldwide. Therefore, these diverse characteristics and factors may create the issue of usability and UX. Furthermore, companies and vendors embed advanced features and functions in smart TV that make the user interface complex and cluttered. Hence, most users are unable to use all the features and functions. The manufacturers have designed several smart TVs with different sizes, user interfaces, and display resolutions. However, the viewing distance, viewing angle, viewing environment (living room, dining hall, room color), and viewing height, brightness, and background color of each smart TV are different from one another [56-59]. These factors can cause various UI issues for viewers [60]. The viewing distance of each viewer varies from one another [56].

Furthermore, each family member has different preferences and characteristics, such as education level, skills, personality, and age [61]. For example, the features and preferences of children vary from adolescence and aged persons. Besides, each age group member may have distinct attributes and preferences. For example, some individuals may enjoy the smart TV from a standard distance, and some may have problems (such as senior citizens with low vision). Similarly, some of them are interested in large font styles, large icons, and the brightness of the screen; the others may not.

3.3. Searching Issues. Smart TV provides various contents from various data sources to the viewers, such as on-demand video, live channels, video and audio, social networking, browsing, gaming, and Web 2.0 features. Most viewers can interact with smart TV by a remote control that makes interaction complex. Besides, all viewers do not have the same skills, knowledge, learnability, and capabilities to interact with a smart TV. Although the electronic program guide (EPGS) may facilitate the viewers to search the programs and channels, however, increasing channels and programs can create searching difficulties on EPGs. Furthermore, each brand's UI structure and layout are different, creating usability, UX, learnability, interactivity, and accessibility in the viewers $[8,12,22,62]$. Also, the room lighting can affect smart TV viewing quality, such as reading, watching, interaction, usability, UX, and learnability. Besides, other factors such as room color and darkness can cause viewing and interactivity problems [63-65]. These parameters can also create eyesight problems such as eyestrain and headaches [66].

3.4. Channel Switching Issues. In contrast to legacy TV, channel searching, switching, and retrieving applications in smart TV are not an easy task. The switching and searching 
of a channel are easy tasks in traditional TV. However, a smart TV has a complex and clutter UI, and many channel switching options and retrieving applications create problems for the viewers [67]. Smart TV supports different interactivity devices such as remote control, mini-keyboard, mouse, gesture, and voice assistant, but the most remote control is the primary interaction device. However, households with a diverse range of users, including kids, technical and nontechnical, house views, senior citizens, and disability viewers, may struggle to retrieve the desired channel in apps [22, 67].

3.5. Clutter Screen Issues. The user interface of various smart TV manufacturers and vendors, such as Android TV, Apple TV, Samsung Smart TV, and LG Smart TV, has designed a cluttered interface and normally created learnability, usability, and accessibility issues. The UI of a smartphone or tablet can easily interact through touch or voice. A smart TV is accessed through remote control from 10 feet, creating interactivity issues. Therefore, instinctively, most people will accept cluttered displays-unorganized and crowded [68]. Also, the apps' focus state, menu, button, links, and elements are necessary for smart TV UI. However, the interactivity from a long viewing distance highlights state issues [68]. Furthermore, smart TV is a shared, lean-back device used by various household members such as kids, children, adults, homemakers, and senior citizens. The perspective, learnability, mental model, age, gender, skills, technical and nontechnical knowledge, and cultural dimension focus and highlight issues for the users.

3.6. Browsing and Scrolling Issues. The browsing of smart TV browsers is a complex task by interacting with remote control and keyboard/mouse due to the clutter UI and interactivity devices. A survey [65] showed that only one of four viewers had used smart TV to browse the online content. Another report showed [69] that most people using a smart TV are an entertainment device for watching video, movie, live channel, and playing games on a big screen. Simultaneously, other functionalities such as social networking, shopping, and maps are rare activities. Browsing in the smart TV browser is a complex task, among other tasks. Each individual's characteristics are different, and the UI of smart is complex [12, 70, 71]. Another issue with a smart TV is the system setting. Smart TV is accessed and watched from 10 feet distance through remote control. The setting of apps or systems or another device configuration is cumbersome for the users in smart TV environments due to clutter user interface, interaction devices, long viewing distance, etc. Due to the watching distance and complex UI of the smart $\mathrm{TV}$, viewers may not perform operations and process as much information on smart TV as they would perform on smartphones or tablets, or computers. The viewing distance and complex user interface have created these types of problems.

3.7. Interaction Device Issue. The conventional remote controls (RCs) have many keys on a small handset to cause three problems. First, keys are generally minor, with the limited size of the handset. Most people have difficulty finding and clicking the correct tickets. Second, the functional information of the keys is overloaded and difficult to remember for the users. Additionally, roughly grouped keys make the problem even worse. Third, a high risk of mode error exists [72]. For example, suppose a user mistakenly presses a function key to alter the video source. In that case, they are automatically redirected from the current workflow to another unknown interface without clarification as to how or why this happened. In addition to these issues, it is difficult to switch between different RCs in multimedia home platforms with multiple RCs when inconsistent and incompatible [73]. Furthermore, such devices have other fundamental structures; secondly, accessing smart TV is complicated due to long-distance viewing and large screen; thirdly, it is complicated to concentrate on the screen element, and fourthly, it is complicated to map between input devices and screen design. Smart TV vendors and companies' technological advancement and proliferation are trying to develop, redesign, and enrich their functions and features of operating system and hardware features to attract viewers and customers and gain market value [15].

\section{Overlooked Factors of Smart TV's UIs}

This section will cover some of the overlooked aspects of the current smart TV user interfaces regarding usability, UX, learnability, accessibility, and cognitive overload. These factors suggest that the smart TV user interface does not take into account various factors such as age, gender, skills, technical and nontechnical, day-to-day feature enrichments, various smart TV UI menu structure and content organization, viewer viewing angle, viewer viewing distance, varying environmental context, and cultural values. These factors are validated by a subjective study conducted for this research.

4.1. Viewers' Age. The first factor affecting the usability and UX of the smart TV user interface is the viewer's age. Smart TVs' existing user interface is a single "one-size-fits-all" user interface that considers all viewers of the same age group. However, the household has different age group viewers with diverse interests, nature, taste, preferences, and needs.

4.2. Interaction Devices. The second factor that affects the usability and UX of the smart TV user interface is the interaction devices. Smart TV is accessed with various interaction devices, including remote controls, tiny keypads, voice, gesture, and smartphone app-based remote controls. However, each device has some pros and cons discussed in the issues and challenges section.

4.3. Viewers' Knowledge and Experience. The third factor that affects the usability and UX of a smart TV user interface is technical skill and knowledge. Usually, smart TV user interfaces have an enriched set of functions and features to provide various services to the viewers and customers; 
however, every viewer may not have the same level of expertise and skills about smart TV's functions and features. Therefore, it creates the problem of usability and UX issues.

4.4. Enrichments of Features and Functions. The fourth factor that affects the usability and UX of smart TV user interfaces is the day-by-day enrichments of features and functionalities in smart TV operating systems, middleware software, and third-party apps. This regular updating and enrichment of parts and functionalities can make the user interface of smart TV clutter and complex for laypeople, children, and senior citizens.

4.5. Different UI Structures. The different menu structures and content organizations are the fifth factors that affect the smart TV user interface's usability and UX. The vendor and companies of smart TV have developed diverse types of UI structures and content organization on a smart TV that creates usability, learnability, and cognitive overload for the users. The sixth factor that affects the usability and UX of the smart TV user interface is the regional, social, and cultural values.

4.6. App-Based Entertainment. The seventh factor that affects the usability and UX of the smart TV user interface is the app-based entertainment on a smart TV. Smart TV provides a diverse source of content and entertainment through app-based to the viewers. The apps of smart TV follow the developing and designing style of smartphones and tablets; however, the interaction behavior, usage behavior, user group, environment, etc., of smart TV are different from smartphone and tablet. Usually, most people use the remote control as an interaction device for smart TV due to the lean-back nature that creates issues in navigation between inter- and intra-apps.

4.7. Viewer's Viewing Angle. Another factor that affects the usability and UX of the smart TV user interface is the viewer's viewing angle in front of the smart TV. Usually, smart TV is accessed through an infrared remote control that needs the correct seating angle for sending commands. Therefore, the viewer may need to sit in front of the smart TV correctly; otherwise, it may create usability and UX.

4.8. Viewer's Viewing Distance. The viewing distance is another factor that affects the usability and UX of the smart TV user interface. The vendors and manufacturers have designed diverse smart TVs with different sizes, display resolutions, video/audio quality, picture quality, and input devices. The viewing requirements for each smart TV differ from one another, such as viewing distance, viewing angle, and environment (living room, dining room, office). These issues create the hurdle of usability and UX in smart TV user interfaces.
4.9. Environmental Context. The environmental variability such as viewing environment (living room, dining hall, room color), viewing height, brightness, and background color of each smart TV is different, affecting the smart TV user interfaces' usability and UX.

\section{Factor Validation}

The watching behavior has been analyzed using a subjective study. The aim was to determine the hidden factors that affect the usability and accessibility of smart TV interfaces. However, UIs may be different for every brand, operating system, and size. However, we confirmed these hidden factors contributing to cognitive overload and complex interfaces.

5.1. Methods and Material. A mixed-mode data collection method was used for collecting responses from the participants. A random sample of 75 viewers was selected from different age groups. This group includes teenagers, homemakers, senior citizens, literate, and educated. The demographics of participants and respective percentages are represented in Table 4.

We asked about 17 questions, of which only two were related to personal information. The responses of 75 respondents were used for the study. The reliability of the questionnaire for 15 items (questions) was tested using SPSS. Cronbach's alpha value of 0.789 shows a reliable and acceptable value (see Table 5).

\section{Results}

The results of the subjective study are presented in the subsections. We used descriptive statistics, i.e., graphs and charts, for presentation. The objectives were to confirm the issues discussed in Section 3.

6.1. Smart TV's Operating System Type. The type of operating systems we found on the viewer's smart TV is shown in Figure 1. The results show that the Android operating system is dominant among all. However, this depends on the region and price of smart TVs. Although the operating systembased smart TV creates interactions, learnability, and cognitive overload, the future is operating system. However, the smart TV needs a more robust operating system than existing operating systems.

6.2. Watching Behavior. TV is enjoyed for watching videos and live channels. For most other tasks, most people use their smartphones. We asked about the watching behavior by asking about the favorite channel type. We received diverse kinds of channels. Although the answer to this question depends on individual interests, we observed that live channels are the primary watching activity on the smart TV. Only a few people, i.e., $5 \%$ of respondents, showed that they sometimes used smart TV for playing games.

It should be noted that young people and kids who received a better response in terms of learnability are better than senior citizens, shown in Figure 2. The method for 
TABLe 4: Demographic information.

\begin{tabular}{lccc}
\hline Participants & Demographics & Number of participants & Percentage \\
\hline \multirow{2}{*}{ Gender } & Female & 18 & 57 \\
& Male & 16 & 76 \\
\hline \multirow{3}{*}{ Age group } & 12 to 20 years & 24 & 21.33 \\
& 20 to 28 years & 25 & 32 \\
& 29 to 37 years & 10 & 13.33 \\
\hline \multirow{2}{*}{ Background } & $>37$ & 67 & 89.33 \\
& Educated & 08 & 10.66 \\
Experience & Literate & 19 & 25.33 \\
& 1 & 17 & 22.66 \\
& 2 & 13 & 17.33 \\
\hline
\end{tabular}

TABLE 5: Cronbach's alpha score for the reliability of the questionnaire.

\begin{tabular}{lcc}
\hline Cronbach's alpha & Cronbach's alpha based on standardized items & No. of items \\
\hline 0.789 & 0.832 & 15
\end{tabular}

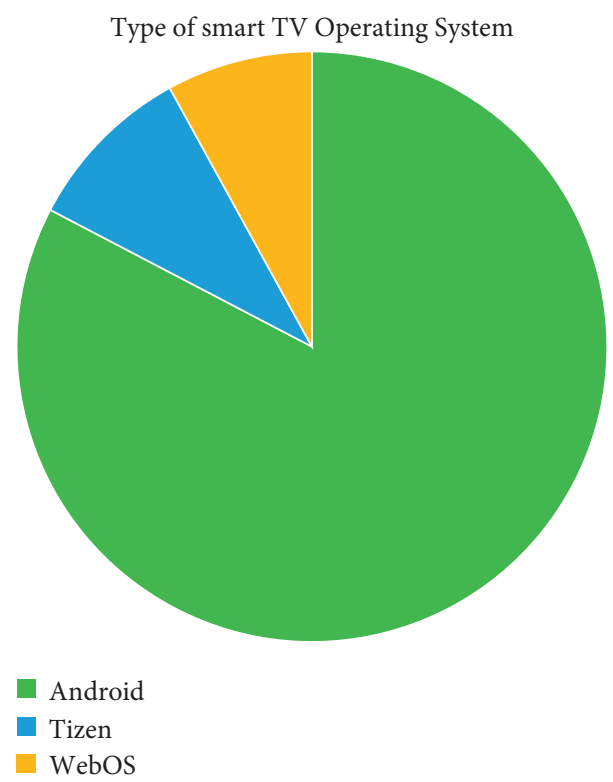

Figure 1: Smart TV OS.

obtaining this score was interviews and observation. We observed and interviewed for the completion of a task. The more easily a task is completed, the learnability curve score will increase. The rating scale score was from 1 to 10 .

6.3. Primary Communication Device with Smart TVs. Besides, a few viewers (5\%) use the app for communication with smart TV; still, the primary device is the common remote control. The communication on the remote control is quite complex [12]. This indicates that the interaction difficulties remain unresolved. The brand-dependent remote controls further create issues in making a standard interaction device for smart TVs. This further shows that besides many smartphones' app-based remote control, the universal communication device is still remote control.
6.4. Navigation Complexity. The results show that navigating among channels and content is complex. The viewers rate 4.8 (average) of 5 difficulty levels, as shown in Figure 3. These difficulties are remote control, complex interface, too many, and irrelevant apps. The difficulty is also due to the clutter interfaces of smart TV. About $93 \%$ of respondents showed that smart TV interfaces are complex.

6.5. Satisfaction Level of Viewers. The viewers use smart TV for various entertainments; however, we received a $91 \%$ dissatisfaction rate from the respondents for the existing UIs. The reasons include a complex interface, traditional remote control, and brand-dependent operating systems. Figure 4 shows the satisfaction level of using smart TV UIs.

6.6. Inter-App Navigational Complexities. As discussed, navigational complexities are among the major challenges for smart TV viewers. The results showed that inter-app navigational complexity is high. We received 4.3 (average) of 5 rates. This figure is high, especially for the low-level experience of smart TV users. The learnability may be increased after some time, but initially, the user faces difficulties searching the desired content on the smart TV.

\section{Discussion}

The results show that the current UIs of various smart TV brands are difficult and create usability and cognitive overload issues. The more the UIs enrich, the more it creates usability issues. The feature enrichment compromises the lean-back nature of smart TV. The viewer's watching behavior shows that they are not satisfied due to interface usability difficulties. The inter-app and intra-app navigational complexity is also a big issue for smart TV due to interaction on the traditional remote control. This study analyzed the overlooked factors and validated these factors using a subjective study. The design and development of the 


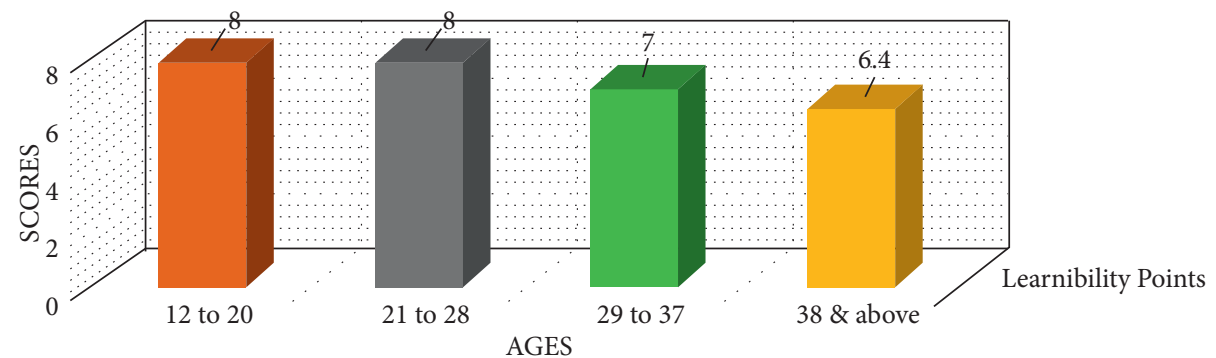

FIgURE 2: Age-wise learnability points.

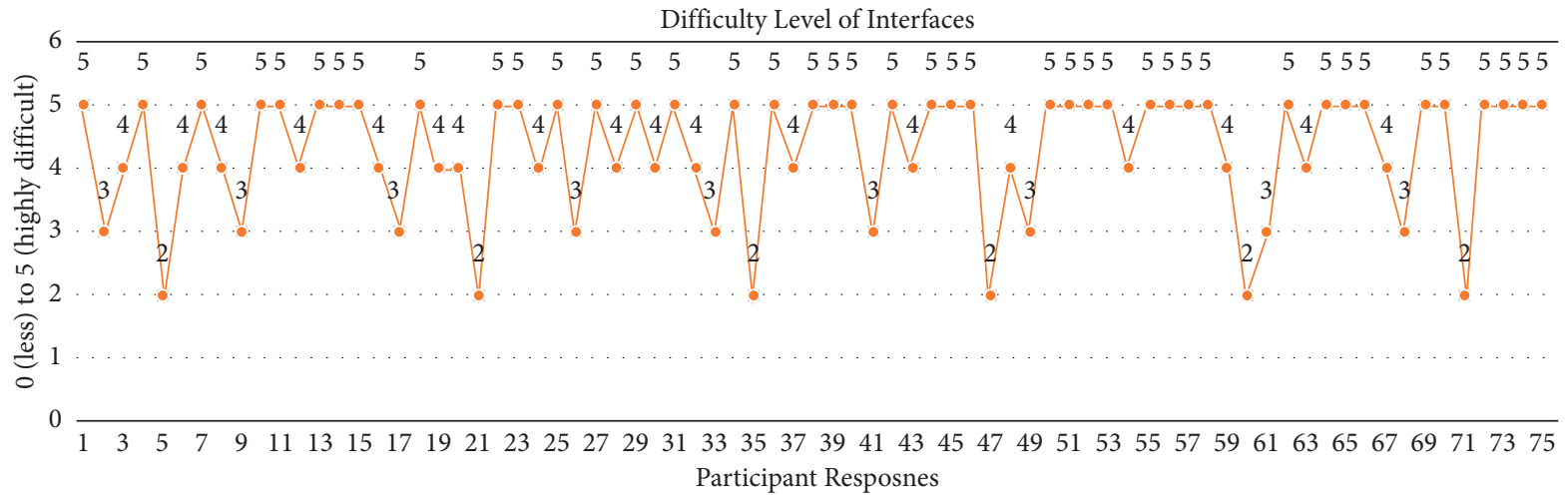

FIgUre 3: Interface difficulty level.

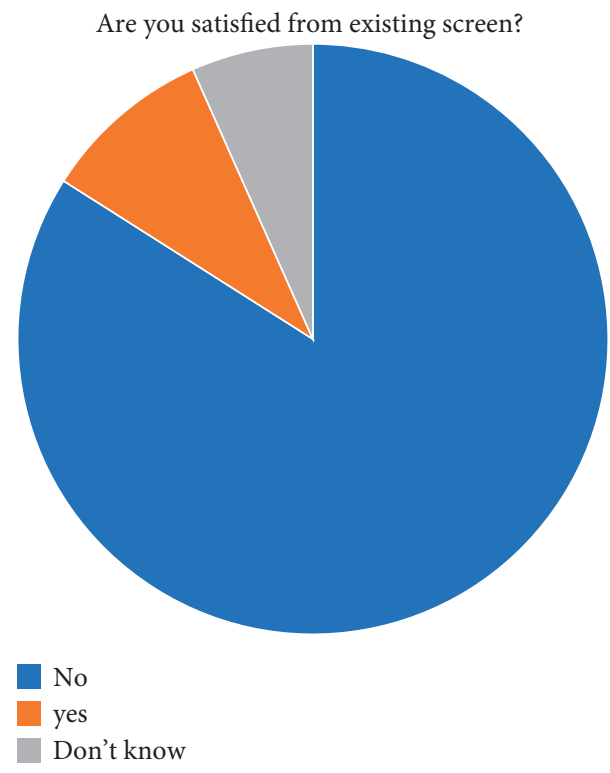

FIgURE 4: Satisfaction level.

smart TV user interface need to understand the viewer's needs, preferences, user models, age, skills, environmental variability, and cultural values. We argue that a personalized adaptive user interface can improve the smart TV's usability, UX, learnability, accessibility, and cognitive overload while performing some operations/actions. The available user interfaces of smart TV are developed based on empirical studies that may not include the characteristics of each household. However, our intended adaptive user interface will use various features and parameters, including implicit and explicit preferences and feedback, interaction styles, watching behaviors, age, gender, usage histories, skills, environmental factors, and facial expression. Besides, an adaptive user interface can play a significant role in smart TV user interfaces and improve the household viewers' usability and UX.

\section{Conclusion and Future Work}

This research study discussed some unnoted factors that affect the usability, UX, learnability, and cognitive overload of the smart TV UI while performing the various essential operations on a smart TV. Future research guidelines and critical factors were also presented to enhance the existing smart TV user interfaces. The results of the subjective study show that existing smart TV user interfaces cannot handle and satisfy the needs and requirements of all household viewers. We argue that dynamically embedding a rich set of smart TV viewers' data (e.g., age, preferences, needs, facial features, generality, skills, cultural values, environmental variability, watching histories, and interaction patterns) in the existing smart TV user interfaces. This integration will enhance usability, UX, learnability and minimize cognitive overload of smart TV viewers. We further argue that designing and developing a smart TV user interface should be treated differently from other tablets or smartphones.

In the future, we aim to expand this work for a personalized adaptive user interface that fulfils the requirements of each viewer and group viewers according to their needs, preferences, age, education, mental model, skills, 
environmental variability to improve the usability, UX, accessibility, and learnability in smart TV viewing scenario. Furthermore, we aim to design and develop an intelligent, personalized adaptive user interface for the smart TV watching environment that adjust layouts, elements, components, font size, color, contrast, theme, app arrangements, menu structure, content, and information presentation according to the viewer's needs, requirements, abilities, experiences, age, mental model, and context. This study suggests that a personalized adaptive user interface can enhance smart TV viewers' usability, UX, accessibility, and learnability.

\section{Data Availability}

The data collected from 75 users in response to the questionnaire and used to support some findings of this study are available from the corresponding author upon request.

\section{Conflicts of Interest}

The authors declare that there are no conflicts of interest regarding the publication of this study.

\section{References}

[1] J. Hussain, A. Ul Hassan, H. S. Muhammad Bilal et al., "Model-based adaptive user interface based on context and user experience evaluation," Journal on Multimodal User Interfaces, vol. 12, no. 1, pp. 1-16, 2018.

[2] I. Alam, S. Khusro, and M. Khan, "Personalized Content Recommendations on Smart TV: Challenges, Opportunities, and Future Research Directions," Entertainment Computing, vol. 38, p. 100418, 2021.

[3] C.-H. Wang and T.-M. Chen, "Incorporating data analytics into design science to predict user intentions to adopt smart TV with consideration of product features," Computer Standards \& Interfaces, vol. 59, pp. 87-95, 2018.

[4] M. Khan, S. Khusro, and I. Alam, "Smart TV-Based Lifelogging Systems: Current Trends, Challenges, and the Road Ahead," Information And Knowledge In Internet of Things, Springer cham, New York, NY, USA, pp. 31-58, 2022.

[5] G. Meixner, F. Paternò, and J. Vanderdonckt, "Past, present, and future of model-based user interface development," $i$-com Zeitschrift für interaktive und kooperative Medien, vol. 10, no. 3, pp. 2-11, 2011.

[6] R a Markets, "Global Smart TVs Market," 2020, https://www. researchandmarkets.com/reports/4908099/global-smart-tvsmarket-forecast-to-2025.

[7] U S B o L Statistics, "American time use survey (ATUS) - 2019 results,” 2020, https://www.bls.gov/news.release/pdf/atus.pdf.

[8] I. Alam, S. Khusro, and M. Naeem, "A review of smart TV: past, present, and future," in Proceedings of the 2017 International Conference on Open Source Systems \& Technologies, pp. 35-41, ICOSST), Lahore, Pakistan, December 2017.

[9] B. S. Ahmed and M. Bures, "EvoCreeper: automated blackbox model generation for smart TV applications," IEEE Transactions on Consumer Electronics, vol. 65, no. 2, pp. 160-169, 2019.

[10] P. C. Hung, K. Kanev, F. Iqbal et al., "A study of children facial recognition for privacy in smart tv," in Proceedings of the International Symposium Computational Modeling of Objects
Represented in Images, pp. 229-240, Niagara Falls, NY, USA, September 2016.

[11] J. Jang and Y. Y. Mun, "Determining and Validating Smart TV UX Factors: A Multiple-Study Approach," International Journal of Human-Computer Studies, vol. 130, no. 5, 2019.

[12] I. Alam, S. Khusro, and M. Khan, "Factors affecting the performance of recommender systems in a smart TV environment," Technologies, vol. 7, no. 2, p. 41, 2019.

[13] P. Seungho, L. Yoojin, and K. Tomimatsu, "Design proposal for smart TV interface and remote controller," International Journal of Asia Digital Art and Design Association, vol. 22, no. 1, pp. 1-13, 2018.

[14] A. Kaya, C. A. Gumussoy, B. Ekmen, and A. E. Bayraktaroglu, "Usability Heuristics for the Set-top Box and TV Interfaces," Human Factors And Ergonomics In Manufacturing \& Service Industries, vol. 31, no. 3, 2021.

[15] I. Alam, S. Khusro, and M. Khan, "Usability barriers in smart TV user interfaces: a review and recommendations," in Proceedings of the 2019 International Conference on Frontiers of Information Technology (FIT), pp. 334-3344, Islamabad, Pakistan, December 2019.

[16] R. Bernhaupt, M. Pirker, and A. Desnos, "The bubble user interface: a tangible representation of information to enhance the user experience in IPTV systems," in Proceedings of the 2014 Companion Publication on Designing Interactive Systems, pp. 85-88, Vancouver, Canada, June 2014.

[17] T. Kim, S. Choi, and H. Bahn, "A personalized interface for supporting multi-users in smart TVs," IEEE Transactions on Consumer Electronics, vol. 62, no. 3, pp. 310-315, 2016.

[18] M. Khan, I. Alam, M. Naeem, and S. Khusro, "There is no such thing as free lunch: an investigation of bloatware effects on smart devices," Journal of Information Communication Technologies and Robotics Applications (JICTRA).(Formally known as NICE Research Journal of Computer Science), vol. 8, pp. 20-30, 2017.

[19] S R. Department, "Smart TV operating system share worldwide in 2018," 2021, https://www.statista.com/statistics/ 882062/worldwide-smart-tv-operating-system-share/.

[20] A. Bruun and J. Stage, "An empirical study of the effects of three think-aloud protocols on identification of usability problems," in Proceedings of the IFIP Conference on HumanComputer Interaction, pp. 159-176, Bamberg, Germany, September 2015.

[21] M. K. Larusdottir, J. Gulliksen, and N. Hallberg, "RAMESFramework supporting user centred evaluation in research and practice," Behaviour \& Information Technology, vol. 38, no. 2, pp. 132-149, 2019.

[22] J. Jang, D. Zhao, W. Hong, Y. Park, and M. Y. Yi, “Uncovering the underlying factors of smart TV UX over time: a multistudy, mixed-method approach," in Proceedings of the ACM International Conference on Interactive Experiences for $T V$ and Online Video, pp. 3-12, Chicago, IL, USA, June 2016.

[23] K. Flaherty, "Smart-TV Usability: Accessing Content is Key," 2015, https://www.nngroup.com/articles/smart-tv-usability/.

[24] N. Choudhary, “Top 6 Key Considerations When Developing Smart TV Application," 2017, https://www.tothenew.com/blog/ top-6-key-considerations-when-developing-smart-tv-application/.

[25] A F Tv, "Design and User Experience Guidelines," 2020, https://developer.amazon.com/docs/fire-tv/design-and-userexperience-guidelines.html.

[26] G Design, “Android TV design guidelines," 2015, https:// designguidelines.withgoogle.com/android-tv/android-tv/ introduction.html. 
[27] A. Ingrosso, V. Volpi, A. Opromolla, E. Sciarretta, and C. M. Medaglia, "UX and usability on smart tv: a case study on a T-commerce application," in Proceedings of the International Conference on HCI in Business, pp. 312-323, Los Angeles, CA, USA, August 2015.

[28] Y. Deng and C. F. Mela, "TV viewing and advertising targeting," Journal of Marketing Research, vol. 55, no. 1, pp. 99-118, 2018.

[29] S. Yoong, G. López, and L. A. Guerrero, "Smart device-based notifications: a survey on user's satisfaction of traditional notification mechanisms," in Proceedings of the International Conference on Applied Human Factors and Ergonomics, pp. 104-114, Los Angeles, CA, USA, July 2017.

[30] M. Sili, M. Garschall, M. Morandell, S. Hanke, and C. Mayer, "Personalization in the user interaction design," in Proceedings of the International Conference on Human-Computer Interaction, pp. 198-207, Toronto, Canada, July 2016.

[31] E. Yigitbas and S. Sauer, "Engineering context-adaptive UIs for task-continuous cross-channel applications," in HumanCentered and Error-Resilient Systems Development, pp. 281300, Springer, New York, NY, USA, 2016.

[32] E. Machado, D. Singh, F. Cruciani et al., "A conceptual framework for adaptive user interfaces for older adults," in Proceedings of the 2018 IEEE International Conference on Pervasive Computing and Communications Workshops, pp. 782-787, PerCom Workshops), Athens, Greece, March 2018.

[33] L. Leventhal, B. Teasley, B. Blumenthal, K. Instone, D. Stone, and M. V. Donskoy, "Assessing user interfaces for diverse user groups: evaluation strategies and defining characteristics," Behaviour \& Information Technology, vol. 15, no. 3, pp. 127-138, 1996.

[34] P. A. Akiki, A. K. Bandara, and Y. Yu, "Engineering adaptive model-driven user interfaces," IEEE Transactions on Software Engineering, vol. 42, no. 12, pp. 1118-1147, 2016.

[35] S. Sarcar, J. Joklnen, A. Oulasvirta, C. Silpasuwanchai, Z. Wang, and X. Ren, "Towards ability-based optimization for aging users," in Proceedings of the International Symposium on Interactive Technology and Ageing Populations, pp. 77-86, Kochi Japan, October 2016.

[36] I. R. M. Association, Application Development and Design: Concepts, Methodologies, Tools, and Applications, IGI Global, Hershey, PA, USA, 2017.

[37] J. S. S. Roy, W. P. Neumann, and D. I. Fels, "User centered design methods and their application in older adult community," in Proceedings of the International Conference on Human Interface and the Management of Information, pp. 462-472, Toronto, Canada, July 2016.

[38] R. Jain, J. Bose, and T. Arif, "Contextual adaptive user interface for Android devices," in Proceedings of the 2013 Annual IEEE India Conference, pp. 1-5, INDICON), Mumbai, India, December 2013.

[39] M. Soui, S. Diab, A. Ouni, A. Essayeh, and M. Abed, "An ontology-based approach for user interface adaptation," in Advances in Intelligent Systems and Computing, pp. 199-215, Springer, New York, NY, USA, 2017.

[40] E. Furtado, V. Furtado, W. B. Silva et al., "An ontology-based method for universal design of user interfaces," in Proceedings of the Workshop on Multiple User Interfaces over the Internet: Engineering and Applications Trends MUI, Lille, France, 2001.

[41] N. Rathnayake, D. Meedeniya, I. Perera, and A. Welivita, "A framework for adaptive user interface generation based on user behavioural patterns," in Proceedings of the 2019 Moratuwa Engineering Research Conference, pp. 698-703, MERCon), Moratuwa, Sri Lanka, July 2019.
[42] J. J. Dudley, J. T. Jacques, and P. O. Kristensson, "Crowdsourcing interface feature design with bayesian optimization," in Proceedings of the 2019 CHI Conference on Human Factors in Computing Systems, pp. 1-12, Glasgow Scotland Uk, May 2019.

[43] M. H. Ramli, A. H. Jantan, A. Kamaruddin, and R. H. Abdullah, "The adaptive model driven approach for enhancing usability of user interface design: a review process," in Proceedings of the Fifth International ACM In-Cooperation HCI and UX Conference, pp. 65-69, Jakarta Surabaya, Bali Indonesia, April 2019.

[44] A. Khan and S. Khusro, "Blind-friendly user interfaces-a pilot study on improving the accessibility of touchscreen interfaces," Multimedia Tools and Applications, vol. 78, no. 13, pp. 17495-17519, 2019.

[45] D. Costa and C. Duarte, "Adapting TV based applications? User interface," in Proceedings of the IADIS International Conference Interfaces and Human Computer Interaction 2012, Lisbon, Portugal, July 2012.

[46] S. V. Kolekar, R. M. Pai, and M. P. Mm, "Rule based adaptive user interface for adaptive E-learning system," Education and Information Technologies, vol. 24, no. 1, pp. 613-641, 2019.

[47] M. Böckle, J. Novak, and M. Bick, "Towards Adaptive Gamification: A Synthesis of Current Developments," in Proceedings of the Twenty Fifth European Conference on Information Systems (ECIS), Guimarães, Portugal, June 2017.

[48] M. Böckle, I. Micheel, M. Bick, and J. Novak, "A design framework for adaptive gamification applications," in Proceedings of the Fifty First Hawaii International Conference on System Sciences, Hilton Waikoloa, HI, USA, January 2018.

[49] B. Bagustari and H. Santoso, "Adaptive user interface of learning management systems for education 4.0: a research perspective," Journal of Physics: Conference Series, vol. 1235, Article ID 12033, 2019.

[50] R. Dias and S. B. i Badia, "AdaptNow-A revamped look for the web: an online web enhancement tool for the elderly," in Proceedings of the International Conference on Computers for Handicapped Persons, pp. 113-120, Paris, France, July 2014.

[51] M. T. Alshammari and A. Qtaish, "Effective adaptive E-learning systems according to learning style and knowledge level," Journal of Information Technology Education, vol. 18, 2019.

[52] A. Khan, S. Khusro, and I. Alam, "Blindsense: an accessibilityinclusive universal user interface for blind people," Engineering, Technology \& Applied Science Research, vol. 8, no. 2, pp. 2775-2784, 2018.

[53] I. Khan and S. Khusro, "Towards the design of context-aware adaptive user interfaces to minimize drivers' distractions," Mobile Information Systems, vol. 2020, Article ID 8858886, 23 pages, 2020.

[54] H. Wu, L. Yang, S. Fu, and X. L. Zhang, "Beyond remote control: exploring natural gesture inputs for smart TV systems," Journal of Ambient Intelligence and Smart Environments, vol. 11, no. 4, pp. 335-354, 2019.

[55] S.-O. Horst, P. C. Murschetz, D. N. Brennan, and M. Friedrichsen, "TV film financing in the era of "connected TV": how do "legacy" broadcasters respond to market changes?" in Handbook of State Aid for Film, pp. 615-633, Springer, New York, NY, USA, 2018.

[56] J. G. Nathan, D. R. Anderson, D. E. Field, and P. Collins, "Television viewing at home: distances and visual angles of children and adults," Human Factors, vol. 27, no. 4, pp. 467-476, 1985.

[57] Tom, "How High Should My TV Be Mounted on Wall?," 2019, https://www.smartaerials.co.uk/blog/how-high-should-my-tvbe-mounted-on-wall. 
[58] samsung, "What size TV do you need for your room," 2020, https://www.samsung.com/us/support/answer/ANS00043948/.

[59] Sony, "What is the recommended height when mounting the TV on a wall," 2019, https://www.sony.com/electronics/ support/articles/00026357.

[60] S. Kindig, “TV sizes and viewing distance," 2020, https://www. crutchfield.com/S-uMxBJBtb1BL/learn/learningcenter/home/ TV_placement.html.

[61] K. Noland and L. Truong, A survey of UK television viewing conditions, Vol. 287, BBC Research \& Development White Paper, , Salford, UK, 2015.

[62] J.-W. Jeong and D.-H. Lee, "Inferring search intents from remote control movement patterns: a new content search method for smart TV," IEEE Transactions on Consumer Electronics, vol. 60, no. 1, pp. 92-98, 2014.

[63] H.-J. Kim, S.-J. Woo, and H. Kim, "Experimental study on living room lighting environment for residential activities," Journal of the Korean Institute of Illuminating and Electrical Installation Engineers, vol. 27, no. 1, pp. 10-16, 2013.

[64] R. Cote, "Reflections on TVs," 2018, https://www.rtings.com/tv/ tests/picture-quality/reflections-screen-finish-matte-and-glossy.

[65] R. Silva, "How does room lighting affect your TV viewing?," 2019, https://www.lifewire.com/room-lighting-and-tvviewing-4147828.

[66] Sciencedaily, "New study tests the effects of watching tv in A dark room," 2006, https://www.sciencedaily.com/releases/ 2006/04/060425015643.htm.

[67] C. W. Gritton, "What? s wrong with smart TV?: how to improve user experience," IEEE Consumer Electronics Magazine, vol. 2, no. 4, pp. 40-43, 2013.

[68] P. Potvin, "Rethinking user interface design for the TV platform," 2016, https://www.toptal.com/designers/ui/tv-uidesign.

[69] E. Perakakis and G. Ghinea, "Smart enough for the web? A responsive web design approach to enhancing the user web browsing experience on smart TVs," IEEE Transactions on Human-Machine Systems, vol. 47, no. 6, pp. 860-872, 2017.

[70] N. Soskic, N. Vranic, and N. Lukic, "Improving User Experience with Unique Channel List on Hybrid Set-Top Boxes," in Proceedings of the 2015 IEEE First International Workshop on Consumer Electronics (CE WS), pp. 46-47, Novi Sad, Serbia, March 2015.

[71] H.-K. Hong and D.-H. Lee, “A personalized refinement technique for semantic multimedia content search in smart TV," IEEE Transactions on Consumer Electronics, vol. 61, no. 4, pp. 581-587, 2015.

[72] C f E i U Design, "Ensure that the remote control is as simple as possible, given the required functionality," 2019, https:// universaldesign.ie/technology-ict/archive-irish-national-itaccessibility-guidelines/digital-tv-equipment-and-services/ guidelines-for-digital-tv-equipment-and-services/remotecontrols/ensure-that-the-remote-control-is-as-simple-aspossible-given-the-required-functionality/.

[73] J. Nielsen, "Remote Control Anarchy," 2004, https://www. nngroup.com/articles/remote-control-anarchy/. 